\title{
Graphene integer quantum Hall effect in the ferromagnetic and paramagnetic regimes
}

\author{
Jason Alicea ${ }^{1}$ and Matthew P. A. Fisher ${ }^{2}$ \\ ${ }^{1}$ Physics Department, University of California, Santa Barbara, California 93106, USA \\ ${ }^{2}$ Kavli Institute for Theoretical Physics, University of California, Santa Barbara, California 93106, USA
}

(Received 3 May 2006; published 23 August 2006)

\begin{abstract}
Starting from the graphene lattice tight-binding Hamiltonian with an on-site $U$ and long-range Coulomb repulsion, we derive an interacting continuum Dirac theory governing the low-energy behavior of graphene in an applied magnetic field. Initially, we consider a clean graphene system within this effective theory and explore integer quantum Hall ferromagnetism stabilized by exchange from the long-range Coulomb repulsion. We study in detail the ground state and excitations at $\nu=0$ and $\nu= \pm 1$, taking into account small symmetrybreaking terms that arise from the lattice-scale interactions, and also explore the ground states selected at $\nu$ $= \pm 3, \pm 4$, and \pm 5 . We argue that the ferromagnetic regime may not yet be realized in current experimental samples, which at the above filling factors perhaps remain paramagnetic due to strong disorder. In an attempt to access the latter regime where the role of exchange is strongly suppressed by disorder, we apply Hartree theory to study the effects of interactions. Here, we find that Zeeman splitting together with symmetry-breaking interactions can in principle produce integer quantum Hall states in a paramagnetic system at $\nu=0, \pm 1$, and \pm 4 , but not at $\nu= \pm 3$ or \pm 5 , consistent with recent experiments in high magnetic fields. We make predictions for the activation energies in these quantum Hall states which will be useful for determining their true origin.
\end{abstract}

DOI: 10.1103/PhysRevB.74.075422

PACS number(s): 73.43.-f, 71.10.Pm

\section{INTRODUCTION}

Recent experimental advances have made possible the isolation of high-quality two-dimensional graphene sheets, ${ }^{1}$ thus opening up a new arena for exploring quantum Hall physics. As illustrated in Fig. 1, graphene is a collection of carbon atoms arranged on a honeycomb lattice. At halffilling and in the absence of a magnetic field, graphene is semimetallic with a Fermi surface consisting of two distinct nodes residing at the Brillouin zone corners. Low-energy excitations about the two nodes are well characterized by two "flavors" of Dirac fermions whose energies obey a linear dispersion relation, $E(\mathbf{k})= \pm \hbar v|\mathbf{k}|$, where for graphene $v$ $\approx 10^{6} \mathrm{~m} / \mathrm{s}^{2,3}$ Applying a magnetic field rearranges the spectrum into Landau levels, each of which is approximately fourfold degenerate near the Fermi level owing to the presence of flavor and spin. The underlying Dirac structure gives rise to an unconventional quantum Hall effect which has been observed experimentally ${ }^{2-5}$ and explored theoretically from numerous perspectives. ${ }^{6-16}$

Initial experiments on the graphene quantum Hall effect reported quantized Hall plateaus at $\sigma_{x y}=\nu e^{2} / h=4(n$ $+1 / 2) e^{2} / h$, where $n$ is an integer. ${ }^{2,3}$ The appearance of these integer quantum Hall states is quite natural from a noninteracting, single-particle perspective, given the approximate fourfold Landau-level degeneracy. There are, however, at least two mechanisms by which additional integer quantum Hall states in graphene may be induced. First, such states can in principle be stabilized by explicit symmetry-breaking fields and/or interactions, the simplest mechanism being Zeeman splitting. In this situation the system would be appropriately characterized as a "quantum Hall paramagnet." Second, provided disorder is sufficiently weak the poorly screened Coulomb interactions in graphene can dramatically modify the single-particle picture, in analogy with the well-studied "quantum Hall ferromagnetism" in GaAs heterostructures. ${ }^{17}$
In the latter system, for instance, exchange interactions strongly stabilize the integer quantum Hall state at $\nu=1$, even in the limit of vanishing Zeeman coupling - that is, the ground state is a ferromagnet. Generally speaking, this mechanism is actually far more common than the first since the Coulomb energy scale is typically much larger than the energy scale associated with symmetry-breaking terms such as the Zeeman energy. Additional exchange-driven integer quantum Hall states can similarly be expected to appear in a sufficiently clean graphene system. In fact, quantum Hall ferromagnetism in graphene promises to be even richer than in GaAs due to the additional flavor degree of freedom, which as we will discuss offers the interesting possibility of

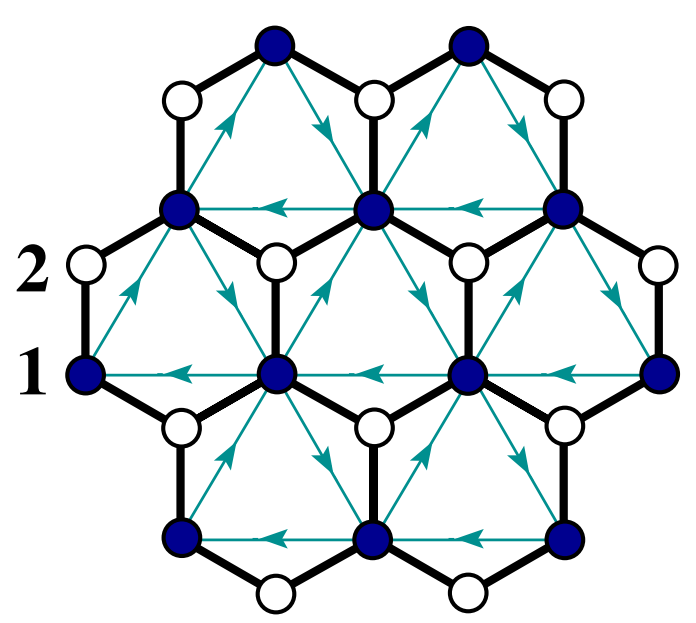

FIG. 1. (Color online) Schematic lattice-scale order in the $\nu$ $=-1$ integer quantum Hall state. "1" and "2" label the two sublattices of the honeycomb. Electrons in the highest-occupied Landau level reside only on sublattice 1 (for instance) and undergo circulating currents around second-neighbor plaquettes oriented along the arrows. 
lattice-scale order coexisting with the integer quantum Hall effect. The problem is thus more analogous to quantum Hall bilayer and $\mathrm{Si}$ metal-oxide-semiconductor field-effect transistor (MOSFET) physics, ${ }^{18}$ where layer and valley indices play a role similar to flavor in graphene.

Interestingly, more recent experiments utilizing higher magnetic fields have in fact resolved additional quantized Hall plateaus at filling factors $\nu=0, \pm 1$, and $\pm 4 .{ }^{5}$ The activation energy measured at $\nu= \pm 4$ was found, somewhat surprisingly, to be dominated by the single-particle Zeeman energy, suggesting that at these filling factors the system resides in the paramagnetic regime due to strong disorder. The origin of the quantum Hall states at $\nu=0$ and $\nu= \pm 1$ is unclear at present and presents an interesting puzzle. In a very recent paper Nomura and MacDonald suggest that these states are due to the onset of quantum Hall ferromagnetism. ${ }^{15}$ Given the apparent spin-splitting origin of the states at $\nu$ $= \pm 4$, however, it is perhaps worth exploring an alternative scenario where the quantum Hall states at $\nu=0$ and $\nu= \pm 1$ are similarly due to explicit symmetry breaking, rather than Coulomb exchange.

The purpose of the present paper is twofold. In the first part, we carry out a detailed exploration of quantum Hall ferromagnetism, focusing on a clean graphene sample. Following this analysis, we consider a dirty system and ask whether symmetry-breaking terms can stabilize integer quantum Hall states in the paramagnetic regime. To this end, we start with the lattice Hamiltonian including on-site $U$ and long-range Coulomb interactions, and derive a continuum interacting Dirac formulation suitable for studying these phenomena. In addition to the usual long-range Coulomb repulsion, we retain shorter-range terms that arise from the microscopic interactions and explicitly break the flavor degeneracy exhibited in a noninteracting theory. Such symmetrybreaking terms have not been taken into account previously and are important for analyzing the physics both in the ferromagnetic and paramagnetic limits. In the ferromagnetic regime, we analyze the ground state and excitations within this theory at integer filling factors in the lowest two Landau levels. At filling factors $\nu= \pm 1$, we show that interactions favor "easy-axis" flavor polarization, leading to a ground state exhibiting charge density wave order on the lattice scale. The $\nu=0$ ground state is found to depend on the strength of interactions relative to the Zeeman coupling and will either be a uniform spin-polarized state or a spin-singlet exhibiting the same lattice-scale structure as at $\nu= \pm 1$. We calculate the spin-wave and particle-hole excitation energies at these filling factors, as well as identify the relevant Skyrmions which are expected to set the transport gap. Interestingly, interactions favor "easy-plane" flavor polarization at $\nu= \pm 3$ and \pm 5 , leading to a ground state that spontaneously breaks U(1) flavor symmetry. Thus, a finite-temperature Kosterlitz-Thouless transition can be expected at these filling factors. Finally, a uniform, spin-polarized ground state is expected at $\nu= \pm 4$. We establish that Skyrmions continue to provide the minimum-energy charge excitations at $\nu= \pm 3$, \pm 4 , and \pm 5 as well, at least in the absence of anisotropy terms.

To explore the paramagnetic regime in a dirty system, we use the expectation that the effects of exchange should be strongly suppressed by disorder and incorporate interactions within Hartree theory. Here, we argue that in principle interactions can give rise to quantum Hall states at $\nu=0, \pm 1$, and \pm 4 , but not at $\nu= \pm 3$ or \pm 5 , which is consistent with recent high-field experiments. We provide estimates for the activation energies in these additional quantum Hall states, which will be useful for determining experimentally whether the $\nu=0$ and \pm 1 states originate from explicit symmetry breaking or quantum Hall ferromagnetism.

\section{CONTINUUM INTERACTING THEORY}

We start with the zero-field lattice Hamiltonian for graphene written as

$$
H=H_{t}+H_{U}+H_{\text {Coul }} \text {, }
$$

where $H_{t}$ describes hopping of electrons across nearestneighbor honeycomb sites,

$$
H_{t}=-t \sum_{\left\langle\mathbf{x x}^{\prime}\right\rangle} \sum_{\alpha=\uparrow, \downarrow}\left[c_{\alpha \mathbf{x}}^{\dagger} c_{\alpha \mathbf{x}^{\prime}}+\text { H.c. }\right],
$$

and $H_{U}$ and $H_{\text {Coul }}$ contain the on-site repulsion and longrange Coulomb interaction, respectively:

$$
\begin{gathered}
H_{U}=U \sum_{\mathbf{x}}\left[\frac{1}{4}\left(n_{\mathbf{x}}\right)^{2}-\frac{1}{3} \mathbf{S}(\mathbf{x})^{2}\right], \\
H_{\text {Coul }}=\frac{1}{2} \sum_{\mathbf{x} \neq \mathbf{x}^{\prime}} V\left(\mathbf{x}-\mathbf{x}^{\prime}\right) n_{\mathbf{x}^{\prime}} n_{\mathbf{x}^{\prime}} .
\end{gathered}
$$

Here $n_{\mathbf{x}}=n_{\uparrow \mathbf{X}}+n_{\downarrow \mathbf{x}}$ is the electron number operator and $\mathbf{S}(\mathbf{x})$ $=\frac{1}{2} c_{\alpha \mathbf{x}}^{\dagger} \boldsymbol{\sigma}_{\alpha \beta} c_{\beta \mathbf{x}}$ is the usual spin operator with $\boldsymbol{\sigma}$ a vector of Pauli matrices. The Coulomb potential is $V(\mathbf{x})=\frac{e^{2}}{4 \pi \epsilon} \frac{1}{|\mathbf{x}|}$, with $\epsilon$ an appropriately chosen dielectric constant (see below). We will introduce the magnetic field upon entering the continuum, which is justified since for experimental field ranges the magnetic length $\ell_{B}=\sqrt{\hbar /(e B)}$ is much larger than the lattice spacing $a_{0}$.

As mentioned above, with one electron per site, the honeycomb band structure exhibits two gapless Dirac points at the Fermi energy, occurring at wave vectors $\pm \mathbf{Q}$ $= \pm(4 \pi / 3,0)$. Focusing on the linearly dispersing excitations near the two nodes, the Fourier-transformed lattice fermion operators may be conveniently expanded in terms of two flavors of continuum Dirac fermion fields (denoted $R$ and $L$ ) as follows:

$$
\begin{gathered}
c_{\alpha \mathbf{q}+\mathbf{Q} a} \sim \gamma \psi_{\alpha R a}(\mathbf{q}), \\
c_{\alpha \mathbf{q}-\mathbf{Q} a} \sim \gamma i \eta_{a b}^{y} \psi_{\alpha L b}(\mathbf{q}) .
\end{gathered}
$$

Here and below we reserve the indices $\alpha$ and $\beta$ for spin, $A$ and $B$ for flavor, and $a$ and $b$ for the honeycomb sublattice. Moreover, $\sigma_{\alpha \beta}^{j}, \tau_{A B}^{j}$, and $\eta_{a b}^{j}$ denote Pauli matrices that contract with the spin, flavor, and sublattice indices, respectively. [Note that according to Eqs. (5) and (6), $\psi_{R 1}$ and $\psi_{L 2}$ correspond to sublattice 1 , while $\psi_{R 2}$ and $\psi_{L 1}$ correspond to sublattice 2.] We will use the convention that suppressed 
indices on the fields are implicitly summed (i.e., $\psi^{\dagger} \psi$ $\left.\equiv \Sigma_{\alpha A a} \psi_{\alpha A a}^{\dagger} \psi_{\alpha A a}\right)$. The normalization on the right-hand side of Eqs. (5) and (6) is chosen to be $\gamma=\sqrt{2} /\left(3^{1 / 4} L\right)$ so that the continuum fields satisfy the canonical anticommutation relations $\left\{\psi_{\alpha A a}(\mathbf{q}), \psi_{\beta B b}^{\dagger}\left(\mathbf{q}^{\prime}\right)\right\}=\delta_{\alpha \beta} \delta_{A B} \delta_{a b}(2 \pi)^{2} \delta^{2}\left(\mathbf{q}-\mathbf{q}^{\prime}\right)$. Realspace Dirac fields are defined according to

$$
\psi_{\alpha A a}(\mathbf{q})=\int d^{2} \mathbf{x} e^{-i \mathbf{q} \cdot \mathbf{x}} \psi_{\alpha A a}(\mathbf{x})
$$

and satisfy $\left\{\psi_{\alpha A a}(\mathbf{x}), \psi_{\beta B b}^{\dagger}\left(\mathbf{x}^{\prime}\right)\right\}=\delta_{\alpha \beta} \delta_{A B} \delta_{a b} \delta^{2}\left(\mathbf{x}-\mathbf{x}^{\prime}\right)$. Note that consistency requires $\psi(\mathbf{x})$ to be a slowly varying field or, equivalently, that $\psi(\mathbf{q})$ be nonzero only within some momentum-space cutoff $\Lambda$. The lattice electron number operator $n_{\mathrm{x} a}$ for sublattice $a$ can now be expressed in terms of real-space Dirac fields as follows:

$$
\begin{aligned}
& n_{\mathbf{x} 1}=\frac{\sqrt{3} a_{0}^{2}}{2}\left[\rho_{R 1}+\rho_{L 2}+e^{2 i \mathbf{Q} \cdot \mathbf{x}} J_{+}^{\dagger}+e^{-2 i \mathbf{Q} \cdot \mathbf{x}} J_{+}\right], \\
& n_{\mathbf{x} 2}=\frac{\sqrt{3} a_{0}^{2}}{2}\left[\rho_{R 2}+\rho_{L 1}-e^{2 i \mathbf{Q} \cdot \mathbf{x}} J_{-}^{\dagger}-e^{-2 i \mathbf{Q} \cdot \mathbf{x}} J_{-}\right],
\end{aligned}
$$

where $\rho_{A a}=\Sigma_{\alpha=\uparrow, \downarrow} \psi_{\alpha A a}^{\dagger} \psi_{\alpha A a}$ contains uniform pieces of the density and $J_{+}=\psi_{R 1}^{\dagger} \psi_{L 2}$ and $J_{-}=\psi_{R 2}^{\dagger} \psi_{L 1}$ contain oscillatory components of the density. The spin operators on each sublattice take on similar forms, with $\frac{1}{2} \boldsymbol{\sigma}$ inserted between each $\psi^{\dagger}$ and $\psi$ above. It follows that in the continuum, the uniform part of the total density is $\rho_{\text {tot }}=\psi^{\dagger} \psi$, while the uniform spin density is $\boldsymbol{S}_{\mathrm{tot}}=\frac{1}{2} \psi^{\dagger} \boldsymbol{\sigma} \psi$.

Using the continuum expansion above and turning on the external magnetic field, we arrive at the desired continuum Hamiltonian, which we write as

$$
\mathcal{H}=\mathcal{H}_{0}+\mathcal{H}_{1} \text {. }
$$

Here $\mathcal{H}_{0}$ denotes the part of the Hamiltonian that is invariant under global $\mathrm{SU}(4)=\mathrm{SU}(2)_{\text {spin }} \times \mathrm{SU}(2)_{\text {flavor }}$ rotations,

$$
\begin{aligned}
\mathcal{H}_{0}= & -i \hbar v \int d^{2} \mathbf{x} \psi^{\dagger}\left[\eta^{x} D_{x}+\eta^{y} D_{y}\right] \psi \\
& +\frac{1}{2} \int d^{2} \mathbf{x} d^{2} \mathbf{x}^{\prime} \rho_{\text {tot }}(\mathbf{x}) V\left(\mathbf{x}-\mathbf{x}^{\prime}\right) \rho_{\mathrm{tot}}\left(\mathbf{x}^{\prime}\right),
\end{aligned}
$$

with $D_{j}=\partial_{j}-i(e / \hbar) A_{j}$. $\mathcal{H}_{1}$ encodes the remaining anisotropy terms which break this $\mathrm{SU}(4)$ symmetry down to $\mathrm{U}(1)_{\text {spin }}$ $\times\left[\mathrm{U}(1) \times \mathrm{Z}_{2}\right]_{\text {flavor }}$,

$$
\begin{aligned}
\mathcal{H}_{1}= & \int d^{2} \mathbf{x}\left[-g \mu_{B} \mathbf{B} \cdot \mathbf{S}_{\mathrm{tot}}+\frac{1}{4} u_{0}\left(\rho_{\mathrm{tot}}^{2}+\rho_{\mathrm{stag}}^{2}\right.\right. \\
& \left.-\frac{8}{3}\left[\mathbf{S}_{R 1}^{2}+\mathbf{S}_{L 2}^{2}+6 \mathbf{S}_{R 1} \cdot \mathbf{S}_{L 2}+(1 \leftrightarrow 2)\right]\right) \\
& \left.-\sum_{\mathbf{r}} v_{1}(\mathbf{r}) \rho_{\mathrm{stag}}(\mathbf{x}+\mathbf{r}) \rho_{\mathrm{stag}}(\mathbf{x})-u_{2}\left[J_{+}^{\dagger} J_{+}+J_{-}^{\dagger} J_{-}^{\dagger}\right]\right],
\end{aligned}
$$

where $\rho_{\text {stag }}=\psi^{\dagger} \tau^{z} \eta^{z} \psi$ represents the staggered electron density between sublattices 1 and 2 of the honeycomb and
$\mathbf{S}_{A a}=\frac{1}{2} \psi_{A a}^{\dagger} \boldsymbol{\sigma} \psi_{A a}$. The sum in Eq. (12) is over triangular lattice vectors $\mathbf{r}=m \mathbf{e}_{+}+n \mathbf{e}_{-}$, where $m, n$ are integers and $\mathbf{e}_{ \pm}=\left(a_{0} / 2\right)( \pm 1, \sqrt{3})$. The first two lines of Eq. (12) contain the Zeeman coupling and the continuum form of the on-site $U$, with

$$
u_{0}=\sqrt{3} a_{0}^{2} U / 4 .
$$

For concreteness we take the magnetic field $\mathbf{B}$ perpendicular to the graphene plane along the $+\hat{\mathbf{z}}$ direction throughout. Apart from the long-range density-density repulsion exhibited in $\mathcal{H}_{0}$, the lattice Coulomb interaction also gives rise to the shorter-range terms in the last two lines of Eq. (12), reflecting lattice-scale physics. The $v_{1}$ term represents an intersublattice repulsion that reflects the smaller Coulomb energy cost for electrons residing on the same sublattice versus opposite sublattices. The $u_{2}$ term represents the intrasublattice repulsion between oscillating components of the density in Eqs. (8) and (9). The coupling constants for these terms are given by

$$
\begin{gathered}
v_{1}(\mathbf{r})=\frac{\sqrt{3} a_{0}^{2}}{8}\left[V(\mathbf{r}+1 / \sqrt{3} \hat{\mathbf{y}})-\left(1-\delta_{\mathbf{r}, 0}\right) V(\mathbf{r})\right], \\
u_{2}=-\sum_{\mathbf{r} \neq 0} \frac{\sqrt{3} a_{0}^{2}}{2} V(\mathbf{r}) \cos \mathbf{Q} \cdot \mathbf{r} \approx \frac{4}{3} a_{0}^{2}\left(\frac{e^{2}}{4 \pi \epsilon a_{0}}\right) .
\end{gathered}
$$

For later use we note that

$$
u_{1} \equiv \sum_{\mathbf{r}} v_{1}(\mathbf{r}) \approx \frac{\sqrt{3}}{4} u_{2} .
$$

Some comments are worthwhile here. The above continuum formulation is expected to remain appropriate out to a cutoff $\Lambda \sim \pi /\left(4 a_{0}\right)$ or so from the Dirac nodes, which constitutes an appreciable fraction of the Brillouin zone. Elimination of modes outside of this radius will renormalize the parameters in the theory somewhat, though for simplicity we have retained the bare values for the coupling constants. Furthermore, other symmetry-allowed interactions will in principle be generated as well; however, we shall assume these are subdominant. We also remark that in Eq. (12) we have only retained terms arising from the lattice Coulomb repulsion that are nonvanishing upon assuming a local form in the continuum (namely, $v_{1}$ and $u_{2}$ ); other terms are expected to essentially average out and will thus be unimportant. Given that $\psi$ is a slowly varying field, it is tempting to approximate $v_{1}$ as a local interaction (as we have done for $u_{2}$ ). For most purposes this is in fact adequate, and we shall often make this assumption to simplify our analysis. However, as we will discuss below, despite appearances assuming a purely local form of $v_{1}$ leaves the $\mathrm{SU}(2)$ flavor symmetry unbroken in the ferromagnetic ground state at filling factors $\nu= \pm 1$, which is why we have retained the finite range of this interaction.

We now turn to screening in the Dirac theory. Since the $v_{1}$ and $v_{2}$ terms are effective only on short length scales, these interactions are essentially unmodified by screening, and we thus assume that the bare dielectric constant for graphene appears in Eqs. (14) and (15). With air on one side of the graphene plane and $\mathrm{SiO}_{2}$ on the other, the unscreened dielec- 
tric constant is estimated to be $\epsilon \approx\left[\left(\epsilon_{+}^{-1}+\epsilon_{-}^{-1}\right) / 2\right]^{-1} \approx 1.6 \epsilon_{0}$, where $\epsilon_{+} \approx \epsilon_{0}$ and $\epsilon_{-} \approx 4 \epsilon_{0}$ correspond to air and $\mathrm{SiO}_{2}$, respectively. The long-range part of the Coulomb interaction in $\mathcal{H}_{0}$ is, however, expected to be (weakly) screened. To estimate the screening, we first note that the presence of the applied magnetic field is expected to become important on length scales longer than roughly the magnetic length, which is much larger than the inverse cutoff $\Lambda^{-1}$. Thus the screened dielectric constant for the zero-field case should not be dramatically modified by the presence of the field. We therefore use the zero-field screened dielectric constant for the longrange Coulomb repulsion in Eq. (11), obtained within the random phase approximation ${ }^{19}$ which yields $\epsilon_{\mathrm{RPA}} \approx \epsilon+e^{2}$ / $(8 \hbar v) \approx 5 \epsilon_{0}$.

\section{OVERVIEW OF DIRAC LANDAU LEVELS}

Before analyzing the full interacting theory, it will be useful to briefly recall the Landau level spectrum for the noninteracting case. ${ }^{6,20}$ Throughout the paper we implicitly work in the symmetric gauge. As a consequence of the Dirac structure, the Landau-level energies in the noninteracting theory are given by

$$
E_{\uparrow \uparrow, n}=\mp \frac{1}{2} g \mu_{B} B+\operatorname{sgn}(n) \sqrt{2 e \hbar v^{2} B|n|},
$$

where $n$ is an integer. Zeeman coupling weakly spin splits the Landau levels, leaving a twofold flavor degeneracy in the absence of interactions. The two-component wave function for spin $\alpha$ and flavor $A$ in the $n$th Landau level can be written

$$
\Phi_{\alpha A}^{n}=\left[\begin{array}{c}
\phi^{n} \\
\chi^{n}
\end{array}\right] .
$$

For $n \neq 0$, the elements $\phi^{n}$ and $\chi^{n}$ are simply wave functions for Schrödinger-equation Landau levels with indices $|n|-1$ and $|n|$, respectively. In the symmetric gauge, the elements are related by $\chi^{n}=|n|^{-1 / 2} \operatorname{sgn}(n) a^{\dagger} \phi^{n}$, where $a^{\dagger}$ is the usual raising operator for Schrödinger-equation Landau levels. One can show that the total probability weight is divided equally between the upper and lower elements of the wave function; in other words, an electron with wave function $\Phi_{\alpha A}^{n \neq 0}$ is equally likely to be found on either honeycomb sublattice. In contrast, the $n=0$ Landau-level wave functions have $\phi^{n=0}=0$, while $\chi^{n=0}$ is a Schrödinger-equation lowestLandau-level wave function. By examining Eqs. (5) and (6) we see that $\Phi_{\alpha R}^{n=0}$ has weight only on sublattice 2 of the honeycomb, while $\Phi_{\alpha L}^{n=0}$ has weight only on sublattice 1 . This qualitative distinction between the $n=0$ and $n \neq 0$ Landaulevel wave functions has important consequences when we incorporate interactions below.

We note that this feature of the $n=0$ Landau-level wave functions is robust against introducing spin-orbit coupling, which admits a term $\mathcal{H}_{S O}=\Delta_{S O} \int d^{2} \mathbf{x} \psi^{\dagger} \eta^{z} \sigma^{z} \psi$ to the Hamiltonian. ${ }^{21}$ In contrast, in the presence of this term the probability density for $n \neq 0$ Landau-level wave functions will no longer be evenly distributed between both sublattices. This correction is quite small, as the induced density differ- ence between the sublattices will be proportional to $\Delta_{S O} \ell_{B} /(\hbar v) \ll 1$ and will hereafter be neglected.

\section{INTEGER QUANTUM HALL FERROMAGNETISM}

In this section we focus on a clean graphene system at integer filling factors and explore "quantum Hall ferromagnetism" using the interacting theory derived above. We will first analyze the $n=0$ Landau level and then turn our attention to the $n=1$ Landau level. In the last part of this section we briefly discuss current experimental relevance for our results.

\section{A. $n=0$ Landau level}

We will employ two simplifying assumptions in our analysis of quantum Hall ferromagnetism in the $n=0$ Landau level. First, we make the standard approximation of ignoring Landau-level mixing and project out states away from the $n=0$ Landau level. This is reasonable given that the spacing between the $n=0$ and $n= \pm 1$ Landau levels is roughly $420 \sqrt{B[\mathrm{~T}]} \mathrm{K}$, where $B[\mathrm{~T}]$ is the magnetic field in teslas, while the Coulomb energy scale

$$
\mathcal{E}_{C} \equiv \frac{e^{2}}{4 \pi \epsilon_{\mathrm{RPA}} \ell_{B}} \approx 130 \sqrt{B[\mathrm{~T}]} \mathrm{K}
$$

is around 3 times smaller. Second, since the noninteracting wave functions reside on only one sublattice or the other, we will set $\psi_{\alpha R / L 1} \rightarrow 0$. With the electron kinetic energy quenched, $\mathcal{H}_{0}$ then becomes

$$
\mathcal{H}_{0}^{n=0}=\frac{1}{2} \int d^{2} \mathbf{x} d^{2} \mathbf{x}^{\prime} \rho_{\text {tot }}(\mathbf{x}) V\left(\mathbf{x}-\mathbf{x}^{\prime}\right) \rho_{\mathrm{tot}}\left(\mathbf{x}^{\prime}\right) .
$$

The anisotropy terms in $\mathcal{H}_{1}$ are dramatically simplified upon projection,

$$
\begin{aligned}
\mathcal{H}_{1}^{n=0}= & \int d^{2} \mathbf{x}\left[-g \mu_{B} \mathbf{B} \cdot \mathbf{S}_{\mathrm{tot}}+\frac{u_{0}}{2} \sum_{A=R / L}\left(\rho_{A 2}^{2}-\frac{4}{3} \mathbf{S}_{A 2}^{2}\right)\right. \\
& \left.-\sum_{\mathbf{r}} v_{1}(\mathbf{r}) \rho_{\mathrm{stag}}(\mathbf{x}+\mathbf{r}) \rho_{\mathrm{stag}}(\mathbf{x})\right]
\end{aligned}
$$

In the projected subspace, $\rho_{\text {tot }}=\rho_{L 2}+\rho_{R 2}, \mathbf{S}_{\text {tot }}=\mathbf{S}_{L 2}+\mathbf{S}_{R 2}$, and $\rho_{\text {stag }}=\rho_{L 2}-\rho_{R 2}$. Note that the $u_{2}$ interaction in Eq. (12) has dropped out altogether.

In the $\mathrm{SU}(4)$-invariant limit with $\mathcal{H}_{1}^{n=0}=0$, despite the fourfold degeneracy of the $n=0$ Landau level the system is an exchange ferromagnet at filling factors $\nu=-1,0$, and +1 (corresponding to a quarter-filled, half-filled, and threequarter-filled levels) and exhibits a quantized Hall conductance $\sigma_{x y}=\nu e^{2} / h$. The $\mathrm{SU}(4)$ spin-flavor symmetry is broken spontaneously in the absence of anisotropy terms, though in accordance with the Mermin-Wagner theorem ${ }^{22}$ true longrange order can exist only at zero temperature. This spontaneous symmetry breaking gives rise to three gapless Goldstone modes at $\nu= \pm 1$ and four at $\nu=0$. These SU(4) "spin waves" have the following dispersion at small momentum: ${ }^{23}$

$$
E_{0}(\mathbf{q})=\kappa\left(\ell_{b} q\right)^{2},
$$

where the stiffness is given by 


$$
\kappa=\frac{1}{4} \sqrt{\frac{\pi}{2}} \mathcal{E}_{C} .
$$

The lowest-energy charge excitations in these integer quantum Hall states are known to be SU(4) Skyrmions, ${ }^{17,24,25}$ whose energies are determined by the stiffness $\kappa$. These topological excitations were explored in Ref. 23. Due to the Coulomb repulsion the minimum-energy Skyrmions are infinitely large and the energy cost for creating a Skyrmion-antiSkyrmion pair is

$$
E_{s k l a s k}=2 \kappa,
$$

which is half that for an electron-hole pair.

The goal of the remainder of this subsection will be to address how this picture is modified by anisotropy terms in $\mathcal{H}_{1}^{n=0}$. In particular, we will discuss the nature of the ground states selected by these symmetry-breaking terms and examine their effects on excitations out of the resulting ordered states.

\section{Filling factors $\nu= \pm 1$}

Consider now the $\nu=-1$ state, corresponding to a quarterfilled $n=0$ Landau level $(\nu=+1$ is related by particle-hole symmetry and will not be discussed separately). Zeeman coupling favors a ground state occupied solely by spin-up electrons. Moreover, the $v_{1}$ term provides an "easy-axis" flavor symmetry that favors having $\left\langle\rho_{\text {stag }}\right\rangle \neq 0$ by occupying either all flavor- $R$ or all flavor- $L$ states. It follows that in the presence of interactions the $\nu=-1$ ground state exhibits a spontaneously broken $Z_{2}$ flavor symmetry and can therefore sustain long-range order at finite temperature. Filling, say, the flavor- $R$ states, the ground state can be written

$$
|\nu=-1\rangle=\prod_{m} c_{\uparrow R, m}^{\dagger}|\mathrm{vac}\rangle,
$$

where $|\mathrm{vac}\rangle$ is the fermion vacuum and $c_{\alpha A, m}^{\dagger}$ adds an electron in the $n=0$ Landau level with spin $\alpha$, flavor $A$, and angular momentum $m$. Since flavors $L$ and $R$ correspond to sublattices 1 and 2 in the projected subspace, the $\nu=-1$ ground state is a spin-polarized charge density wave (CDW), having a larger electron density on one of the two honeycomb sublattices. The charge order is illustrated schematically in Fig. 1. Physically, this CDW arises because electrons can remain farther apart by occupying one sublattice before the other, thereby minimizing their Coulomb energy. We emphasize, however, that only the relatively small number of electrons participating in the $n=0$ Landau level (roughly 1.4 $\times 10^{-5} B[\mathrm{~T}]$ electrons per hexagon) contribute to this order, which might make it difficult to observe with a scanning tunneling microscope (STM). Another experimental signature of the CDW at $\nu=-1$ may come from carbon NMR measurements. First, there is a net electron spin only on the higher-density sublattice, producing a magnetic moment per site of roughly $1.4 \times 10^{-5} B[\mathrm{~T}] \mu_{B}$ with which the (relatively rare) carbon atoms with nuclear spin can interact. A second effect arises from more subtle lattice-scale structure that exists in the $\nu=-1$ state. Indeed, it follows from Eqs. (5) and (6) that electrons on the higher-density sublattice participate in currents circulating around second-neighbor plaquettes, oriented along the arrows in Fig. 1. This circulation in turn gives rise to a magnetic moment along the applied field direction that couples to atoms in the lower-density sublattice. Using our zero-field relation between the lattice and continuum fields, we crudely estimate the magnitude of this induced moment to be $5 \times 10^{-5} B[\mathrm{~T}] \mu_{B}$. These effects should induce a small splitting in the nuclear spin precession frequencies for carbon atoms on the two sublattices, which may be measurable.

We turn next to excitations in the presence of anisotropy terms. The three Goldstone modes present in the SU(4)invariant limit become gapped. These generalized "spinwave" gaps contain important information regarding the stability of the spin and CDW order in the ground state, as well as for the transport gap. Employing the usual lowest-Landaulevel projection, the energies for these three branches can be obtained essentially exactly. We follow closely the approach in the review in Ref. 17, generalized to the SU(4) case. The procedure is straightforward though tedious, and here we shall only outline the calculation. Define

$$
S^{\mu \nu}=\frac{1}{2} \psi^{\dagger} \tau^{\mu} \sigma^{\nu} \psi
$$

where $\mu$ and $\nu$ run over $0, x, y$, and $z$ and $\tau^{0}$ and $\sigma^{0}$ are identity matrices. We wish to calculate the energies of $\overline{S^{0 x}}(\mathbf{q})|\nu=-1\rangle, \overline{S^{x 0}}(\mathbf{q})|\nu=-1\rangle$, and $\overline{S^{x x}}(\mathbf{q})|\nu=-1\rangle$, which turn out to be exact eigenstates of the projected interacting Hamiltonian. Here the overbar indicates a lowest-Landaulevel projection, $\overline{S^{0 x}}(\mathbf{q})$ creates a spin wave with momentum $\mathbf{q}, \overline{S^{x 0}}(\mathbf{q})$ creates a "flavor wave," and $\overline{S^{x x}}(\mathbf{q})$ creates a mixed spin-flavor wave. The energies of these excited states are conveniently calculated in first quantization. The Hamiltonian in momentum space becomes

$$
\begin{aligned}
\mathcal{H}_{0}^{n=0}=\frac{1}{2} \int_{\mathbf{k}} V(\mathbf{k}) \bar{\rho}_{\text {tot }}(\mathbf{k}) \bar{\rho}_{\text {tot }}(-\mathbf{k}), \\
\mathcal{H}_{1}^{n=0}=-g \mu_{B} B \overline{S^{0 z}}(\mathbf{k}=0)+\frac{1}{4} u_{0} \int_{\mathbf{k}}\left(\bar{\rho}_{\text {tot }}(\mathbf{k}) \bar{\rho}_{\text {tot }}(-\mathbf{k})\right. \\
+4 \overline{S^{z 0}}(\mathbf{k}) \overline{S^{z 0}}(-\mathbf{k})-\frac{4}{3}\left[\overline{S^{z i}}(\mathbf{k}) \overline{S^{z i}}(-\mathbf{k})\right. \\
\left.\left.+\overline{S^{0 i}}(\mathbf{k}) \overline{S^{0 i}}(-\mathbf{k})\right]\right)-4 \sum_{\mathbf{r}} v_{1}(\mathbf{r}) \int_{\mathbf{k}} e^{i \mathbf{k} \cdot \mathbf{r} \overline{S^{z 0}}(\mathbf{k}) \overline{S^{z 0}}(-\mathbf{k}),}
\end{aligned}
$$

where $i$ is summed over $x, y$, and $z$. The projected first quantized operators are given explicitly by

$$
\begin{gathered}
\bar{\rho}_{\text {tot }}(\mathbf{k})=e^{-\left(\ell_{B} k\right)^{2} / 4} \sum_{j} p_{\mathbf{k}}(j), \\
\overline{S^{\mu \nu}}(\mathbf{k})=\frac{1}{2} e^{-\left(\ell_{B} k\right)^{2} / 4} \sum_{j} p_{\mathbf{k}}(j) \tau^{\mu}(j) \sigma^{\nu}(j),
\end{gathered}
$$

where $j$ runs over each electron in the ground state and $p_{\mathbf{k}}(j)$ is a unitary operator satisfying 


$$
\begin{gathered}
p_{\mathbf{k}}(j) p_{\mathbf{q}}(j)=e^{i\left(\ell_{B}^{2} / 2\right) k \wedge q} p_{\mathbf{k}+\mathbf{q}}(j), \\
{\left[p_{\mathbf{k}}(i), p_{\mathbf{q}}(j)\right]=2 i \delta_{i j} \sin \left(\ell_{B}^{2} k \wedge q / 2\right) p_{\mathbf{k}+\mathbf{q}}(j),}
\end{gathered}
$$

with $k \wedge q=\hat{\mathbf{z}} \cdot(\mathbf{k} \times \mathbf{q})$. Evaluating the commutators $\left[\mathcal{H}_{0}^{n=0}+\mathcal{H}_{1}^{n=0}, \overline{S^{0 x}}(\mathbf{q})\right]$, etc., on the ground state yields the corresponding excitation energies.

At small momentum, we obtain the following energies for spin-wave, flavor-wave, and mixed spin-flavor-wave excitations, respectively:

$$
\begin{gathered}
E_{\sigma}(\mathbf{q}) \approx g \mu_{B} B+\kappa_{\sigma}\left(\ell_{B} q\right)^{2}, \\
E_{f}(\mathbf{q}) \approx \sqrt{\frac{\pi^{3}}{24}}\left(\frac{a_{0}}{\ell_{B}}\right) u_{1} \rho_{0}+\kappa_{f}\left(\ell_{B} q\right)^{2}, \\
E_{\sigma f}(\mathbf{q})=g \mu_{B} B+E_{f}(\mathbf{q}),
\end{gathered}
$$

where $\rho_{0}=1 /\left(2 \pi \ell_{B}^{2}\right)$ and the stiffnesses are

$$
\begin{gathered}
\kappa_{\sigma}=\left(u_{0}-u_{1}\right) \rho_{0}+\kappa, \\
\kappa_{f}=u_{1} \rho_{0}+\kappa .
\end{gathered}
$$

At large momentum $|\mathbf{q}| \rightarrow \infty$, these excitations correspond to the three types of separated particle-hole excitations out of the ferromagnetic state (particle-hole excitations can be created by flipping a spin, flavor, or both). Their energies are

$$
\begin{gathered}
E_{\sigma}(\infty)=g \mu_{B} B+2\left(u_{0}-u_{1}\right) \rho_{0}+4 \kappa, \\
E_{f}(\infty)=2 u_{1} \rho_{0}+4 \kappa, \\
E_{\sigma f}(\infty)=g \mu_{B} B+2 u_{1} \rho_{0}+4 \kappa .
\end{gathered}
$$

The most noteworthy feature of these excitation energies is the small value of the gap for flavor-wave excitations, which is $E_{f}(0) \approx 4 \times 10^{-3}(B[T])^{3 / 2} \mathrm{~K}$. In fact, had we ignored the finite-range of $v_{1}$ here, we would incorrectly conclude that the flavor-wave gap vanishes. Assuming a local form, the sublattice repulsion becomes proportional to $\overline{S^{z 0}}(\mathbf{x})^{2}$, which is simply a constant when acting on a uniform density states in the quarter-filled Landau level. The lack of symmetry breaking from such local interactions has been noted previously in studies of quantum Hall bilayers. ${ }^{26}$ As a result the flavor-wave gap is down by an additional factor of $a_{0} / \ell_{B}$ from what one might naively anticipate. Nevertheless, longrange CDW order is expected to persist up to a transition temperature of order $\kappa_{f} / \ln \left[\kappa_{f} / E_{f}(0)\right] .{ }^{27}$ The spin order is comparatively more robust, as the spin-wave gap is $g \mu_{B} B$ $\sim B[\mathrm{~T}] \mathrm{K},{ }^{5}$ which for typical field ranges is around 50 times larger or so than the flavor wave gap.

The smallness of the flavor-wave gap has important implications for Skyrmions as well, which will continue to set the charge gap. In particular, Skyrmions will be cheaper to create solely in flavor space, leaving the spin order intact. The competition between the sublattice repulsion $v_{1}$, which favors small Skyrmions, and the long-range Coulomb, which favors infinite Skyrmions, will set a length scale for the minimum-energy Skyrmions. However, the ratio of the flavor-wave gap to the Coulomb energy scale $\mathcal{E}_{C}$ is quite small, given approximately by $3 \times 10^{-5} B[\mathrm{~T}]$. For comparison, the ratio of the Zeeman gap to the Coulomb energy in GaAs is roughly $6 \times 10^{-3} \sqrt{B[\mathrm{~T}]},{ }^{28}$ which at $10 \mathrm{~T}$ is around 60 times larger. Hence, corrections to the energies of flavor Skyrmions arising from the nonzero flavor-wave gap are expected to be small. Approximating their energies by those for infinite Skyrmions in the absence of a flavor-wave gap, the activation energy arising from flavor Skyrmions is estimated to be $\Delta_{\nu=-1} \approx \kappa_{f}$. ${ }^{24}$ These results imply that the CDW order will diminish extremely rapidly as one tunes away from $\nu$ $=-1$, whereas the spin order will be much more robust. One possible test of these predictions would be to measure the activation energy at constant perpendicular magnetic field with varying in-plane fields. ${ }^{29}$ The activation energy arising from flavor Skyrmions, being independent of spin, will be insensitive to changes in the latter.

\section{Filling factor $\nu=0$}

The ground state at $\nu=0$, corresponding to a half-filled $n=0$ Landau level, will depend on the strength of the Zeeman coupling and on-site $U$ relative to the sublattice repulsion $v_{1}$. If the former terms dominate, the ground state will be a spin-polarized flavor-singlet

$$
|\nu=0\rangle_{\mathrm{sp}}=\prod_{m} c_{\uparrow R, m}^{\dagger} c_{\uparrow L, m}^{\dagger}|\mathrm{vac}\rangle,
$$

which in contrast to $\nu=-1$ respects all graphene lattice symmetries. If the sublattice repulsion dominates, however, the ground state will be a flavor-polarized spin-singlet

$$
|\nu=0\rangle_{\mathrm{fp}}=\prod_{m} c_{\uparrow R, m}^{\dagger} c_{\downarrow R, m}^{\dagger}|\mathrm{vac}\rangle,
$$

and thus also exhibit the lattice-scale CDW order illustrated in Fig. 1. Comparing the energies of these two states, one finds that the ground state will be spin polarized if $g \mu_{B} B$ $+2 u_{0} \rho_{0}>4 u_{1} \rho_{0}$, while a flavor-polarized ground state emerges if $g \mu_{B} B+2 u_{0} \rho_{0}<4 u_{1} \rho_{0}$. According to Eqs. (13) and (16), we have $u_{0} \rho_{0} \approx 0.08 U[\mathrm{eV}] B[\mathrm{~T}] \mathrm{K}$ and $u_{1} \rho_{0}$ $\approx 0.4 B[\mathrm{~T}] \mathrm{K}$, where $U[\mathrm{eV}]$ is the strength of the on-site repulsion evaluated in electron volts $(\mathrm{eV})$. Ascertaining which scenario prevails is complicated by the rather large uncertainty in the value of the on-site $U$. Previous estimates have suggested $U \sim 5-12 \mathrm{eV},{ }^{30}$ though here a slightly lower range of values may be appropriate due to the presence of the $\mathrm{SiO}_{2}$ substrate. With $g=1.4$, which is the smallest value reported in recent high-field experiments, ${ }^{5}$ the critical value for $U$ above which the ground state is spin polarized is estimated to be $U_{c} \sim 4 \mathrm{eV}$. Hence we cannot conclusively determine whether the ground state is spin polarized, though this regime can always be reached by increasing the effective Zeeman coupling via the introduction of an in-plane magnetic field component. Below we will discuss excitations in both regimes.

Consider first the situation where the ground state is spin polarized. Here there are four low-lying branches of excitations, generated by $\overline{S^{x x}}(\mathbf{q}) \pm i \overline{S^{y x}}(\mathbf{q})$ and $\overline{S^{0 x}}(\mathbf{q}) \pm \overline{S^{z x}}(\mathbf{q})$, which create spin waves by breaking flavor singlets as required by 
the Pauli principle. Schematically, the former breaks singlets by sending $(|R\rangle|L\rangle-|L\rangle|R\rangle) \rightarrow|R\rangle|R\rangle$ (or $|L\rangle|L\rangle)$, while the latter sends $(|R\rangle|L\rangle-|L\rangle|R\rangle) \rightarrow|R\rangle|L\rangle$ (or $|L\rangle|R\rangle$ ). That is, $\overline{S^{x x}}(\mathbf{q}) \pm i \overline{S^{y x}}(\mathbf{q})$ creates a spin wave by doubly occupying a given flavor, while $\overline{S^{0 x}}(\mathbf{q}) \pm \overline{S^{z x}}(\mathbf{q})$ does not. Proceeding as in $\nu=-1$, at small momentum the respective energies for the first and second pairs of spin waves are

$$
\begin{gathered}
E_{1 \pm}^{\mathrm{sp}}(\mathbf{q}) \approx g \mu_{B} B+2 u_{0} \rho_{0}-4 u_{1} \rho_{0}+\kappa_{f}\left(\ell_{B} q\right)^{2}, \\
E_{2 \pm}^{\mathrm{sp}}(\mathbf{q}) \approx g \mu_{B} B+\kappa_{\sigma}\left(\ell_{B} q\right)^{2},
\end{gathered}
$$

while at $|\mathbf{q}| \rightarrow \infty$ we have

$$
E_{1,2 \pm}^{\mathrm{sp}}(\infty)=g \mu_{B} B+2 u_{0} \rho_{0}-2 u_{1} \rho_{0}+4 \kappa .
$$

The stiffnesses $\kappa_{f}$ and $\kappa_{\sigma}$ are the same as at $\nu=-1$. Note that having $E_{1 \pm}^{\mathrm{sp}}(0)>0$ requires that $g \mu_{B} B+2 u_{0} \rho_{0}>4 u_{1} \rho_{0}$, which is the same condition given above for realizing a spinpolarized ground state.

We will assume that $u_{0}-2 u_{1}<0$, implying that $E_{1 \pm}^{\mathrm{sp}}(0)$ $<E_{2 \pm}^{\mathrm{sp}}(0)$. This occurs for $U \leq 10 \mathrm{eV}$, which we believe is reasonable. Due to the smaller gap for exciting spin waves by doubly occupying a given flavor, it follows that the charge gap will be set by mixed spin-flavor-textured Skyrmions. Unfortunately, the large uncertainty in the strength of the on-site $U$ does not permit us to make a quantitative estimate for the activation energy set by these Skyrmions. We can at least say qualitatively that as one tunes away from $\nu=0$ they should induce a depletion in the total spin of the system along with a simultaneous revival of the sublattice CDW order. How rapidly this occurs will of course depend on the optimal Skyrmion size, which we are unable to determine.

Consider now the case where the sublattice repulsion dominates, leading to a flavor-polarized ground state. There are again four low-lying branches of excitations, generated by $\overline{S^{x x}}(\mathbf{q}) \pm i \overline{S^{x y}}(\mathbf{q})$ and $\overline{S^{x 0}}(\mathbf{q}) \pm \overline{S^{x z}}(\mathbf{q})$, which create flavor waves by breaking spin singlets. The first pair of operators break singlets by sending $(|\uparrow\rangle|\downarrow\rangle-|\downarrow\rangle|\uparrow\rangle) \rightarrow|\uparrow\rangle|\uparrow\rangle \quad$ (or $|\downarrow\rangle|\downarrow\rangle)$, while the latter operators send $(|\uparrow\rangle|\downarrow\rangle-|\downarrow\rangle|\uparrow\rangle)$ $\rightarrow|\uparrow\rangle|\downarrow\rangle$ (or $|\downarrow\rangle|\uparrow\rangle)$. The energies for the first and second pairs of flavor waves are

$$
\begin{gathered}
E_{1 \pm}^{\mathrm{fp}}(\mathbf{q}) \approx \mp g \mu_{B} B+2\left(2 u_{1}-u_{0}\right) \rho_{0}+\kappa_{f}\left(\ell_{B} q\right)^{2}, \\
E_{2 \pm}^{\mathrm{fp}}(\mathbf{q}) \approx 2\left(2 u_{1}-u_{0}\right) \rho_{0}+\kappa_{f}\left(\ell_{B} q\right)^{2},
\end{gathered}
$$

at small momentum, while

$$
\begin{gathered}
E_{1 \pm}^{\mathrm{fp}}(\infty)=\mp g \mu_{B} B+2\left(3 u_{1}-u_{0}\right) \rho_{0}+4 \kappa, \\
E_{2 \pm}^{\mathrm{fp}}(\infty)=2\left(3 u_{1}-u_{0}\right) \rho_{0}+4 \kappa .
\end{gathered}
$$

Here the gap is smallest for flavor waves exciting by doubly occupying spin-up states as favored by Zeeman coupling, so the charge gap will be set by mixed spin-flavor-textured Skyrmions in this case as well. Away from $\nu=0$ such Skyrmions will produce a rapid depletion of the CDW order and revival of spin polarization.

Distinguishing experimentally between these two ground states can be achieved by measuring the activation energy in the presence of an in-plane magnetic field that enhances the effective $g$ factor. If the ground state is spin polarized, then the activation energy should increase with the in-plane field since the larger $g$ factor favors smaller (and thus higherenergy) Skyrmions. On the other hand, if the ground state is flavor polarized, a decrease in the activation would be expected-at least initially, before the condition for having a flavor-polarized ground state is violated. Here, the enhanced $g$ factor reduces the energy cost for slowly varying spinflavor fluctuations according to Eq. (45), leading to an increase in optimal Skyrmion size and hence a reduction in the optimal Skyrmion energy.

\section{B. $n=1$ Landau level}

Next, we will discuss quantum Hall ferromagnetism at $\nu$ $=3,4$, and 5, corresponding to a quarter-filled, half-filled, and three-quarter-filled $n=1$ Landau level. ( $n=-1$ is related by particle-hole symmetry.) We will again ignore Landaulevel mixing and project onto the $n=1$ level. Since the wave functions now reside on both sublattices, we must return to the full interacting theory defined by Eqs. (11) and (12).

In the $\mathrm{SU}(4)$-invariant limit with $\mathcal{H}_{1}=0$, the system will again be an exchange ferromagnet at filling factors $\nu=3,4$, and 5. It is interesting to explore the lowest-energy charge excitations here and in particular to ask whether the transport gap is set by topological Skyrmions or separated particlehole excitations. In the case of Schrödinger-equation Landau levels, it is known that Skyrmions constitute the minimumenergy charge excitation only in the lowest Landau level. ${ }^{25}$ Since we are dealing here with two-component wave functions whose elements consist of lowest-Landau-level and next-lowest-Landau level Schrödinger-equation wave functions, the answer in the present case is not a priori obvious.

To address this issue, we calculate the energies of the generalized SU(4) "spin-wave" branches in the SU(4)invariant limit. While the convenient first-quantized formalism described in the previous subsection is no longer at our disposal, these energies can be computed directly using second quantization. For simplicity, let us consider $\nu=3$ and assume the following symmetry-broken ground state:

$$
|0\rangle=\prod_{m} c_{\uparrow R, m}^{\dagger}|\mathrm{vac}\rangle,
$$

where $c_{\uparrow R, m}^{\dagger}$ adds an electron into the $n=1$ Landau level. (Our attention will be restricted to this filling factor since the energies are unchanged at $\nu=4$ and 5.) We evaluate explicitly the energy of spin-wave excitations created by the operator $S_{\sigma}^{-}(\mathbf{q}) \equiv \int_{\mathbf{x}} e^{-i \mathbf{q} \cdot \mathbf{x}}\left[S^{0 x}(\mathbf{x})-i S^{0 y}(\mathbf{x})\right]$, where $S^{0 x}$ and $S^{0 y}$ are defined in Eq. (26). This choice is arbitrary, since which branch we evaluate is immaterial due to the assumed SU(4) symmetry. Upon projecting onto the $n=1$ Landau level, we have $S_{\sigma}^{-}(\mathbf{q}) \rightarrow \overline{S_{\sigma}^{-}}(\mathbf{q})$, with

$$
\begin{aligned}
\overline{S_{\sigma}^{-}}(\mathbf{q})= & \int_{\mathbf{x}} e^{-i \mathbf{q} \cdot \mathbf{x}} \sum_{l, m}\left[\Phi_{l}^{n=1}(\mathbf{x})\right]^{\dagger} \Phi_{m}^{n=1}(\mathbf{x}) \\
& \times\left[c_{\downarrow R, l}^{\dagger} c_{\uparrow R, m}+c_{\downarrow L, l}^{\dagger} c_{\uparrow L, m}\right] .
\end{aligned}
$$

Here $\Phi_{l, m}^{n=1}(\mathbf{x})$ are two-component wave functions defined in 
Eq. (18), with $l$ and $m$ labeling the angular momentum (we have suppressed the spin and flavor indices since the wave functions do not depend on these). A spin wave in the projected space is thus given by $|\mathbf{q}\rangle=\overline{S_{\sigma}^{-}}(\mathbf{q})|0\rangle$ and has an excitation energy

$$
E^{\prime}(\mathbf{q})=\frac{\left\langle\mathbf{q}\left|\overline{\mathcal{H}_{0}}\right| \mathbf{q}\right\rangle}{\langle\mathbf{q} \mid \mathbf{q}\rangle}-\frac{\left\langle 0\left|\overline{\mathcal{H}_{0}}\right| 0\right\rangle}{\langle 0 \mid 0\rangle},
$$

where $\overline{\mathcal{H}_{0}}$ is the $\mathrm{SU}(4)$-invariant part of the Hamiltonian projected onto the $n=1$ Landau level. Letting $L^{2}$ be the system size, we obtain

$$
\begin{aligned}
& E^{\prime}(\mathbf{q})=2 \int_{\mathbf{k}} V(k) \sin ^{2}\left(\ell_{B}^{2} k \wedge q / 2\right) N(k), \\
N(k)= & \frac{2 \pi}{L^{2}} \int_{\mathbf{x y}} e^{i \mathbf{k} \cdot(\mathbf{x}-\mathbf{y})} \sum_{l, m}\left[\Phi_{l}^{n=1}(\mathbf{x})\right]^{\dagger} \Phi_{m}^{n=1}(\mathbf{x}) \\
& \times\left[\Phi_{m}^{n=1}(\mathbf{y})\right]^{\dagger} \Phi_{l}^{n=1}(\mathbf{y})=e^{-\left(\ell_{B} k\right)^{2} / 2}\left[1-\left(\ell_{B} k\right)^{2} / 4\right]^{2} .
\end{aligned}
$$

Note that replacing the wave functions in Eq. (53) by onecomponent Schrödinger-equation Landau-level wave functions reproduces the known spin-wave energies for the latter case. ${ }^{25}$ Thus, Eqs. (52) and (53) constitute a straightforward generalization of the spin-wave energies to the case of twocomponent wave functions.

Equation (52) yields the following energy dispersion at small momentum:

$$
\begin{aligned}
& E_{0}^{\prime}(\mathbf{q}) \approx \kappa^{\prime}\left(\ell_{B} q\right)^{2}, \\
& \kappa^{\prime}=\frac{7}{64} \sqrt{\frac{\pi}{2}} \mathcal{E}_{C} .
\end{aligned}
$$

This implies that a Skyrmion-anti-Skyrmion pair costs an energy

$$
E_{s k / a s k}^{\prime}=2 \kappa^{\prime} .
$$

The energy of a particle-hole excitation meanwhile is given by

$$
E_{p / h}^{\prime}=E_{0}^{\prime}(\infty)=\frac{11}{16} \sqrt{\frac{\pi}{2}} \mathcal{E}_{C} .
$$

Comparing these, we see that $E_{s k / a s k}^{\prime} / E_{p / h}^{\prime}=7 / 22<1$; consequently, in the SU(4)-invariant limit Skyrmions will set the transport gap in the $n=1$ Landau level as well.

\section{Filling factor $\nu=3$}

We will now incorporate the anisotropy terms in $\mathcal{H}_{1}$ to deduce the probable ground states selected by interactions, considering $\nu=3$ first. Due to the Zeeman coupling, we will assume a spin-polarized ground state here. The ordering in flavor space remains to be determined. The most general flavor-polarized state favored by the long-range Coulomb repulsion can be expressed as

$$
|\theta, \phi\rangle=\prod_{m=0}^{\infty}\left[\cos \frac{\theta}{2} c_{\uparrow R, m}^{\dagger}+\sin \frac{\theta}{2} e^{i \phi} c_{\uparrow L, m}^{\dagger}\right]|\mathrm{vac}\rangle .
$$

The angles $\theta$ and $\phi$ specify the polarization direction in flavor space. With the ground state spin polarized, the only terms in $\mathcal{H}_{1}$ remaining that break the flavor degeneracy are the $v_{1}$ and $u_{2}$ interactions. Naively, the former favors a state with $\left\langle\rho_{\text {stag }}\right\rangle \neq 0$, while the latter favors having $\left\langle J_{ \pm}\right\rangle \neq 0$. It is easy to show, however, that both expectation values vanish for any choice of $\theta$ and $\phi$ in Eq. (58). The vanishing of $\left\langle\rho_{\text {stag }}\right\rangle$ follows from the fact that the $n=1$ wave functions carry equal weight on both sublattices. The vanishing of $\left\langle J_{ \pm}\right\rangle$ is a consequence of the fact that the elements $\phi^{n=1}$ and $\chi^{n=1}$ in Eq. (18) are given by wave functions for different Schrödinger-equation Landau levels; hence, $\Sigma_{m}\left[\phi_{m}^{n=1}\right]^{*} \chi_{m}^{n=1}$ $=0$. In other words, as opposed to what we saw at $\nu=-1$, the direct contributions from the interactions leave the flavor symmetry unbroken here.

There is, nevertheless, subtler flavor symmetry breaking by interactions that arises due to exchange. Specifically, $v_{1}$ favors in-plane flavor polarization $(\theta=\pi / 2)$, while $u_{2}$ favors out-of-plane flavor polarization (e.g., $\theta=0$ ). Denoting the projected $v_{1}$ and $u_{2}$ interactions as $\overline{\mathcal{H}_{v_{1}}}$ and $\overline{\mathcal{H}_{u_{2}}}$, respectively, we obtain the following variational energies:

$$
\begin{gathered}
\left\langle\pi / 2, \phi\left|\overline{\mathcal{H}_{v_{1}}}+\overline{\mathcal{H}_{u_{2}}}\right| \pi / 2, \phi\right\rangle=\frac{\rho_{0} L^{2}}{8} u_{2} \rho_{0}, \\
\left\langle 0, \phi\left|\overline{\mathcal{H}_{v_{1}}}+\overline{\mathcal{H}_{u_{2}}}\right| 0, \phi\right\rangle=\frac{\rho_{0} L^{2}}{2} u_{1} \rho_{0} .
\end{gathered}
$$

Since $u_{1} \approx \sqrt{3} u_{2} / 4$, it follows that the in-plane polarized state has lower energy so that the $\nu=3$ ground state is given by

$$
|\nu=3\rangle \sim \prod_{m=0}^{\infty}\left[c_{\uparrow R, m}^{\dagger}+e^{i \phi} c_{\uparrow L, m}^{\dagger}\right]|\mathrm{vac}\rangle,
$$

with a spontaneously chosen in-plane polarization angle $\phi$. This state is characterized by a nonzero order parameter $\left\langle\psi^{\dagger}\left(\tau^{x}+i \tau^{y}\right) \psi\right\rangle \neq 0$ and in general breaks translation, rotation, and reflection lattice symmetries for graphene. Using Eqs. (5) and (6), one can show that this discrete symmetry breaking is due to lattice-scale fermion currents circulating around nearest-neighbor honeycomb plaquettes, with a specific pattern determined by the polarization angle $\phi$.

Since this state spontaneously breaks U(1) flavor symmetry, a finite-temperature Kosterlitz-Thouless transition is expected here. The physics associated with this transition has been examined in detail previously in the context of quantum Hall bilayers, where the layer degree of freedom plays the role of flavor. ${ }^{18,26,31}$ One obstacle for observing such a transition in the bilayer problem is the finite tunneling amplitude between layers, which explicitly breaks the U(1) psuedospin symmetry that is analogous to the U(1) flavor symmetry in our case. Here, however, the U(1) flavor symmetry appears to be more robust. Graphene may therefore eventually provide a clean setting for studying the physics of a Kosterlitz- 
Thouless transition in the quantum Hall effect. Further theoretical exploration of this phenomenon in the present context would certainly be interesting.

\section{Filling factor $\nu=4$}

Similarly to what we found at $\nu=0$, the ground state at $\nu=4$ depends on the strength of Zeeman coupling and the on-site $U$ relative to that of the interactions $v_{1}$ and $u_{2}$. The former terms again favor a spin-polarized flavor-singlet state, while the latter interactions now favor an in-plane flavorpolarized spin-singlet state. Evaluating the variational energies of these states, we find that the spin-polarized state has a lower energy if $g \mu_{B} B+u_{0} \rho_{0}>u_{1} \rho_{0}+u_{2} \rho_{0} / 4$. This inequality is satisfied even with a vanishing on-site $U$ and a $g$ factor as small as unity. Thus we will assume here that $\nu=4$ ground state is spin polarized:

$$
|\nu=4\rangle=\prod_{m} c_{\uparrow R, m}^{\dagger} c_{\uparrow L, m}^{\dagger}|\mathrm{vac}\rangle .
$$

We note, however, that the gaps for spin waves out of this state are positive only if $g \mu_{B} B+2 u_{0} \rho_{0}>2 u_{1} \rho_{0}+u_{2} \rho_{0} / 2$, which provides a slightly more restrictive condition for having a fully spin-polarized ground state. With $g=1.4$, the latter inequality is satisfied provided $U \geqslant 2 \mathrm{eV}$.

\section{Filling factor $\nu=5$}

Finally, the situation at $\nu=5$ is analogous to that at $\nu=3$. The ground state will have all spin-up states in the $n=1$ Landau level occupied to satisfy the Zeeman coupling. Due to the sublattice repulsion $v_{1}$, the remaining spin-down electrons in the ground state are expected to be in-plane flavor polarized:

$$
|\nu=5\rangle \sim \prod_{m}\left[c_{\downarrow R, m}^{\dagger}+e^{i \phi} c_{\downarrow L, m}^{\dagger}\right]|\nu=4\rangle .
$$

This state exhibits the same type of lattice-scale order as the $\nu=3$ ground state and also spontaneously breaks $\mathrm{U}(1)$ flavor symmetry so that a Kosterlitz-Thouless transition is expected here as well.

\section{Experimental relevance}

Initial sightings of the graphene quantum Hall effect observed integer quantum Hall states only at filling factors $\nu$ $= \pm 2, \pm 6, \pm 10$, etc., ${ }^{2,3}$ corresponding to filled nearly fourfolddegenerate Landau levels. Quantum Hall ferromagnetism was thus entirely absent in these experiments. This is almost certainly due to a collapse of the exchange gap responsible for quantum Hall ferromagnetism by strong disorder, a phenomenon that has been established both theoretically $15,32,33$ and experimentally. ${ }^{34,35}$

As discussed in the Introduction, more recent experiments utilizing higher magnetic fields observed additional quantized Hall plateaus at $\nu=0, \pm 1$, and $\pm 4 .{ }^{5}$ A natural question to ask is whether the appearance of these plateaus is a manifestation of quantum Hall ferromagnetism. Nomura and MacDonald, who very recently derived an experimental criterion for realizing quantum Hall ferromagnetism in graphene, sug- gest that this is indeed the case. However, the measured activation energy at $\nu= \pm 4$ was found to be dominated by the single-particle Zeeman splitting, suggesting that here the system is perhaps more appropriately characterized as a paramagnet. At present it is unclear whether the same is true for the plateaus at $\nu=0$ or $\nu= \pm 1$. Measurements of the activation energies there will likely provide some hints as to their origin.

In the following section, we will explore the possibility that the system is indeed paramagnetic at these newly observed quantized Hall plateaus and that their origin is due to explicit symmetry-breaking terms in the Hamiltonian (rather than exchange) as appears to be the case at $\nu= \pm 4$. In an attempt to access this regime, we will assume that the effects of exchange are effectively "canceled" by disorder and utilize Hartree theory to examine the spin and flavor splitting of the $n=0$ and $n=1$ Landau levels by such terms. This analysis will hopefully provide useful input for determining the true nature of the $\nu=0$ and $\nu= \pm 1$ states.

\section{PARAMAGNETIC REGIME}

It will be instructive to begin our analysis of the paramagnetic regime by applying Hartree theory initially in a clean system. Disorder effects will be discussed at the end of this section. Since in our treatment of the ferromagnetic regime we found that the $u_{2}$ interaction dropped out at the $n=0$ Landau level and was effective only through exchange at $n$ $=1$, we will ignore this term here. Doing so will greatly streamline our discussion, and our results do not depend on this simplification. We retain the remaining symmetrybreaking terms in $\mathcal{H}_{1}$ and consider the following interacting Hamiltonian:

$$
\begin{aligned}
\tilde{\mathcal{H}}= & \int d^{2} \mathbf{x}\left\{-i \hbar v \psi^{\dagger}\left[\eta^{x} D_{x}+\eta^{y} D_{y}\right] \psi-g \mu_{B} \mathbf{B} \cdot \mathbf{S}_{\text {tot }}\right. \\
& \left.+u_{0}\left[\rho_{\uparrow, \text { tot }} \rho_{\downarrow, \text { tot }}+\rho_{\uparrow, \text { stag }} \rho_{\downarrow, \text { stag }}\right]-u_{1} \rho_{\text {stag }}^{2}\right\},
\end{aligned}
$$

where $\rho_{\alpha, \text { tot }}$ and $\rho_{\alpha \text {,stag }}$ are the total and staggered densities for spin $\alpha$ (e.g., $\rho_{\uparrow, \text { stag }}=\psi_{\uparrow}^{\dagger} \tau^{z} \eta^{z} \psi_{\uparrow}$ ). We have ignored the finite range of the sublattice repulsion $v_{1}$, as a local form will now be adequate. Furthermore, we have dispensed with the manifestly $\mathrm{SU}(2)_{\mathrm{spin}}$-invariant form of the on-site $U$ term in favor of a form which will be more convenient to work with here. Note also that we have dropped pieces of the on-site $U$ interaction involving $J_{ \pm}$, since again these will be unimportant.

Our aim will be to employ Hartree theory to obtain effective single-particle energy levels for the interacting system and in particular to extract the resulting particle-hole excitation energies which will be of potential relevance for experimentally measured activation energies. To proceed we decouple the interactions by linearizing in fluctuations of the total and staggered densities about their mean values, yielding an effective mean-field Hamiltonian 


$$
\begin{aligned}
\tilde{\mathcal{H}}_{\mathrm{MF}}= & \int d^{2} \mathbf{x}\left\{-i \hbar v \psi^{\dagger}\left[\eta^{x} D_{x}+\eta^{y} D_{y}\right] \psi-g \mu_{B} \mathbf{B} \cdot \mathbf{S}_{\mathrm{tot}}\right. \\
& \left.+\sum_{\alpha=\uparrow, \downarrow}\left[\lambda_{\alpha, \mathrm{t}} \rho_{\alpha, \mathrm{tot}}+\lambda_{\alpha, \mathrm{s}} \rho_{\alpha, \mathrm{stag}}\right]-\mu_{\mathrm{s}} \rho_{\mathrm{stag}}\right\}+E_{\mathrm{const}}
\end{aligned}
$$

The constant-energy term that arises upon decoupling is given by

$$
\begin{aligned}
E_{\text {const }}= & \int d^{2} \mathbf{x}\left\{-u_{0}\left[\left\langle\rho_{\uparrow, \text { tot }}\right\rangle\left\langle\rho_{\downarrow, \text { tot }}\right\rangle+\left\langle\rho_{\uparrow, \text { stag }}\right\rangle\left\langle\rho_{\downarrow, \text { stag }}\right\rangle\right]\right. \\
& \left.+u_{1}\left\langle\rho_{\text {stag }}\right\rangle^{2}\right\} .
\end{aligned}
$$

Furthermore, the new coupling constants appearing in Eq. (65) are defined as follows:

$$
\begin{gathered}
\lambda_{\uparrow / \downarrow, \mathrm{t}}=u_{0}\left\langle\rho_{\downarrow / \uparrow, \text { tot }}\right\rangle, \\
\lambda_{\uparrow / \downarrow, \mathrm{s}}=u_{0}\left\langle\rho_{\downarrow / \uparrow, \mathrm{stag}}\right\rangle, \\
\mu_{\mathrm{s}}=2 u_{1}\left\langle\rho_{\mathrm{stag}}\right\rangle .
\end{gathered}
$$

The $\lambda_{\alpha, \mathrm{t}}$ and $\lambda_{\alpha, \mathrm{S}}$ terms represent effective chemical potentials for the total and staggered densities for spin $\alpha$, while $\mu_{s}$ behaves as an overall staggered chemical potential. Assuming uniform expectation values for the total and staggered densities, it is now straightforward to diagonalize the meanfield Hamiltonian $\mathcal{H}_{\mathrm{MF}}$ to obtain the desired effective singleparticle levels for the interacting theory. Below we examine the resulting level structure at integer filling factors in the $n=0$ and $n=1$ Landau levels.

\section{A. $n=0$ Landau level}

The two-component $n=0$ Landau-level wave functions for flavor $A$ and spin $\alpha$ retain the same form as in the noninteracting case (see Sec. III). Their energies, however, are now shifted as follows:

$$
\begin{aligned}
& E_{\uparrow / \downarrow, R}=\mp \frac{1}{2} g \mu_{B} B+\lambda_{\uparrow / \downarrow, \mathrm{t}}-\lambda_{\uparrow \downarrow \downarrow, \mathrm{s}}+\mu_{s}, \\
& E_{\uparrow \downarrow, L}=\mp \frac{1}{2} g \mu_{B} B+\lambda_{\uparrow \downarrow \downarrow, \mathrm{t}}+\lambda_{\uparrow / \downarrow, \mathrm{s}}-\mu_{s} .
\end{aligned}
$$

In particular, we see that the flavor degeneracy exhibited in the noninteracting case is generically lifted by interactions. Below we specialize to filling factors $\nu= \pm 1$ and $\nu=0$ to obtain the effective level structure in the respective ground states. We will find here that Hartree theory predicts the same ground states as in the ferromagnetic case, due to the fact that direct contributions from interactions rather than exchange selected the ordering in the latter case. Excitation energies, however, which are our main interest, will be dramatically modified by the loss of exchange.

\section{Filling factors $\nu= \pm 1$}

We will again consider only $\nu=-1$ since $\nu=+1$ is related by particle-hole symmetry. The mean-field energy at $\nu=-1$ is
$\Delta_{\mathrm{S}}>\Delta_{u_{1}}$

$\Delta_{\mathrm{s}}<\Delta_{u_{1}}$

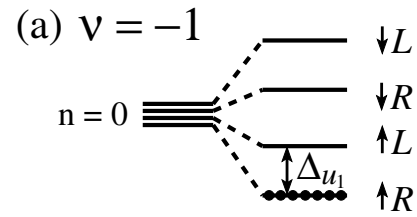

(b) $v=0$
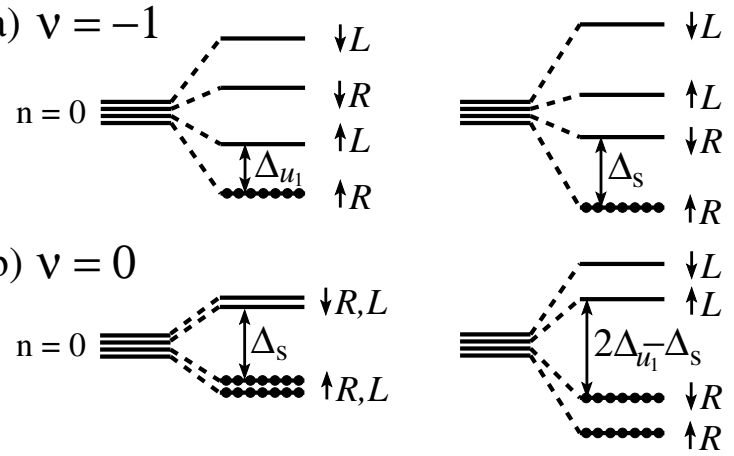

(c) $v=4$

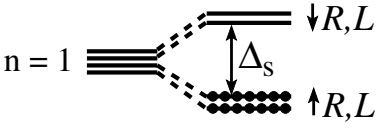

FIG. 2. Schematic effective single-particle energy levels obtained within a Hartree analysis of the interacting theory at (a) $\nu$ $=-1$, (b) $\nu=0$, and (c) $\nu=4$. Here $\Delta_{s}=g \mu_{B} B+2 u_{0} \rho_{0}$, while $\Delta_{u_{1}}$ $=4 u_{1} \rho_{0}$. For $\nu=-1$ and 0 , the levels on the left-side correspond to $\Delta_{s}>\Delta_{u_{1}}$, while the right side corresponds to $\Delta_{s}<\Delta_{u_{1}}$.

minimized by filling all spin-up, flavor- $R$ states (or all spinup, flavor- $L$ states). Setting $\left\langle\rho_{\uparrow, \text { tot }}\right\rangle=-\left\langle\rho_{\uparrow, \text { stag }}\right\rangle=\rho_{0}$ and $\left\langle\rho_{\downarrow \text {,tot }}\right\rangle$

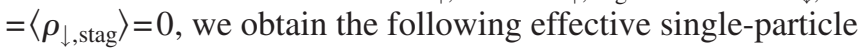
levels:

$$
\begin{gathered}
E_{\uparrow R}=-\frac{1}{2} g \mu_{B} B-2 u_{1} \rho_{0}, \\
E_{\uparrow L}=-\frac{1}{2} g \mu_{B} B+2 u_{1} \rho_{0}, \\
E_{\downarrow R}=+\frac{1}{2} g \mu_{B} B+2 u_{0} \rho_{0}-2 u_{1} \rho_{0}, \\
E_{\downarrow L}=+\frac{1}{2} g \mu_{B} B+2 u_{1} \rho_{0} .
\end{gathered}
$$

By examining Eqs. (72)-(75), we see that the minimum energy required to make a particle-hole excitation out of the ground state is either $4 u_{1} \rho_{0}$ or $g \mu_{B} B+2 u_{0} \rho_{0}$, whichever is smaller. Again we note that from Eqs. (13) and (16) we have $u_{0} \rho_{0} \approx 0.08 U[\mathrm{eV}] B[\mathrm{~T}] \mathrm{K}$ and $u_{1} \rho_{0} \approx 0.4 B[\mathrm{~T}] \mathrm{K}$, where $U[\mathrm{eV}]$ is expected to be on the order of a few electron volts. Figure 2(a) illustrates schematically the effective singleparticle levels in these two cases.

\section{Filling factor $\nu=0$}

Just as we saw in the ferromagnetic regime, the $\nu=0$ ground state here depends on the strength of Zeeman coupling and the on-site $U$ relative to the sublattice repulsion $u_{1}$. Evaluating the mean-field energetics for these states, we again find that the ground state will be spin polarized if $g \mu_{B} B+2 u_{0} \rho_{0}>4 u_{1} \rho_{0}$, while a flavor-polarized ground state 
exhibiting lattice-scale CDW order occurs if $g \mu_{B} B+2 u_{0} \rho_{0}$ $<4 u_{1} \rho_{0}$.

Let us first deal with the case where the ground state is spin polarized. Here, we set $\left\langle\rho_{\uparrow, \text { tot }}\right\rangle=2 \rho_{0}$ and $\left\langle\rho_{\downarrow, \text { tot }}\right\rangle$ $=\left\langle\rho_{\uparrow / \downarrow, \text { stag }}\right\rangle=0$, yielding the following energy levels:

$$
\begin{gathered}
E_{\uparrow R / L}^{\mathrm{sp}}=-\frac{1}{2} g \mu_{B} B, \\
E_{\downarrow R / L}^{\mathrm{sp}}=+\frac{1}{2} g \mu_{B} B+2 u_{0} \rho_{0} .
\end{gathered}
$$

Since all spin-up states are occupied, the energy for a particle-hole excitation is clearly given by $g \mu_{B} B+2 u_{0} \rho_{0}$. Consider alternatively the case where the ground state is flavor polarized, and we have $\left\langle\rho_{\uparrow / \downarrow \text {,tot }}\right\rangle=-\left\langle\rho_{\uparrow / \downarrow \text {,stag }}\right\rangle=\rho_{0}$ corresponding to occupying all flavor $R$ states. The effective single-particle levels are now

$$
\begin{gathered}
E_{\uparrow / \downarrow R}^{\mathrm{fp}}=\mp \frac{1}{2} g \mu_{B} B+2 u_{0} \rho_{0}-4 u_{1} \rho_{0}, \\
E_{\uparrow / \downarrow L}^{\mathrm{fp}}=\mp \frac{1}{2} g \mu_{B} B+4 u_{1} \rho_{0} .
\end{gathered}
$$

Consequently, the minimum energy for a particle-hole excitation is $8 u_{1} \rho_{0}-2 u_{0} \rho_{0}-g \mu_{B} B$. The single-particle levels for both scenarios are illustrated schematically in Fig. 2(b).

\section{B. $n=1$ Landau level}

We will make an additional simplification in our treatment of the $n=1$ Landau level and set $\lambda_{\alpha, \mathrm{s}}=\mu_{\mathrm{s}}=0$ at the outset. This is not necessary and does not affect our results, and we do so only for the sake of simplicity. On physical grounds one expects these terms to vanish given that the noninteracting $n=1$ wave functions carry equal weight on both sublattices. Furthermore, it is easy to show that in any case they do not lead to a splitting of the flavor degeneracy in the $n=1$ Landau level.

With this simplification, the wave functions are unchanged from the noninteracting case, while the energies for flavor $A$ become

$$
E_{\uparrow / \downarrow A}^{\prime}=\mp \frac{1}{2} g \mu_{B} B+\lambda_{\uparrow / \downarrow, \mathrm{t}}+\sqrt{2 e \hbar v^{2} B} .
$$

In contrast to the $n=0$ Landau level, the flavor degeneracy remains unbroken by interactions here. The important physical implication is that there are gapless charge excitations at $\nu=3$ and 5, and thus no quantum Hall effect would be predicted by Hartree theory at these filling factors. This is not too surprising since in our analysis of the ferromagnetic regime we found that interactions broke the flavor degeneracy at $\nu=3$ and 5 only via exchange.

The levels are spin split, however, and there is a gap at $\nu=4$. All spin-up orbitals are occupied in the ground state, so here we have $\left\langle\rho_{\uparrow, \text { tot }}\right\rangle=2 \rho_{0}$ and $\left\langle\rho_{\uparrow, \text { tot }}\right\rangle=0$. The effective single-particle energies are thus given by

$$
\begin{gathered}
E_{\uparrow A}^{\prime}=-\frac{1}{2} g \mu_{B} B+\sqrt{2 e \hbar v^{2} B}, \\
E_{\downarrow A}^{\prime}=+\frac{1}{2} g \mu_{B} B+2 u_{0} \rho_{0}+\sqrt{2 e \hbar v^{2} B},
\end{gathered}
$$

and the particle-hole excitation energy is $g \mu_{B} B+2 u_{0} \rho_{0}$. The energy levels here are illustrated in Fig. 2(c).

\section{Discussion}

To summarize, our disorder-free Hartree analysis suggests that Zeeman coupling together with symmetry-breaking interactions can in principle induce quantum Hall states in a paramagnetic system at filling factors $\nu=0, \pm 1$, and \pm 4 , but not at $\nu= \pm 3$ or \pm 5 . The activation energies at $\nu= \pm 4$ obtained in Hartree theory-corresponding to one-half of the particle-hole energies-are

$$
\Delta_{\nu= \pm 4}=\frac{1}{2} g \mu_{B} B+u_{0} \rho_{0} .
$$

At filling factors $\nu= \pm 1$ and $\nu=0$, the activation energies depend on whether (i) $g \mu_{B} B+2 u_{0} \rho_{0}>4 u_{1} \rho_{0}$ or (ii) $g \mu_{B} B$ $+2 u_{0} \rho_{0}<4 u_{1} \rho_{0}$. In case (i) the activation energies at these filling factors are predicted to be

$$
\begin{gathered}
\Delta_{\nu= \pm 1}^{(i)}=2 u_{1} \rho_{0}, \\
\Delta_{\nu=0}^{(i)}=\frac{1}{2} g \mu_{B} B+u_{0} \rho_{0},
\end{gathered}
$$

while for case (ii) we have

$$
\begin{gathered}
\Delta_{\nu= \pm 1}^{(i i)}=\frac{1}{2} g \mu_{B} B+u_{0} \rho_{0}, \\
\Delta_{\nu=0}^{(i i)}=4 u_{1} \rho_{0}-u_{0} \rho_{0}-\frac{1}{2} g \mu_{B} B .
\end{gathered}
$$

We note that in the second case the activation energy at $\nu$ $=0$ is larger than that for $\nu= \pm 1$ and \pm 4 .

These results will of course be modified by the inclusion of disorder. In particular, one may ask whether the latticescale character of the wave functions, which was essential for the flavor symmetry breaking at $n=0$ and lack thereof at $n=1$, survives upon disordering the system. We expect that microscopic features of the wave functions will indeed remain robust, provided the length scale associated with the disorder is long compared with the lattice spacing. Furthermore, it is clear that assuming uniform expectation values for the total and staggered densities as we did above will not be valid in the presence of disorder. This introduces a considerable degree of complexity, as the mean-field Hamiltonian in this case is not easily diagonalized and must be solved selfconsistently. However, we expect that disorder can be effectively accounted for by a (possibly magnetic field-dependent) broadening of the effective single-particle levels found above, leading to a reduction in the activation energies we predicted by considering a clean system. A more serious 
treatment of disorder and interactions will clearly be desirable, but will not be pursued here.

Even at the crude level of our treatment, it is interesting to compare our predictions from Hartree theory with the highfield experiments mentioned above that resolved additional quantum Hall states at $\nu=0, \pm 1$, and \pm 4 . First, Hartree theory is consistent with the appearance of these quantum Hall states and also can potentially provide a simple explanation for the conspicuous absence of those at $\nu= \pm 3$ and \pm 5 -namely, a lack of flavor symmetry breaking due to the form of the higher-Landau-level wave functions. Second, the activation energy predicted at $\nu= \pm 4$ is given by the Zeeman energy plus a contribution from the on-site $U$ that is linear in the perpendicular magnetic field, the latter being unchanged by the addition of an in-plane magnetic field component [see Eq. (83)]. The measured energy gap for the spin-split Landau levels was similarly found to be given by the Zeeman energy, supplemented by a phenomenological Landau-level broadening varying linearly with the perpendicular magnetic field. ${ }^{5}$ If one interprets this Landau-level broadening as a contribution to the energy splitting arising from the on-site $U$, one obtains $U \sim 2 \mathrm{eV}$, which is of the right order of magnitude. Finally, if $g \mu_{B} B+2 u_{0} \rho_{0}<4 u_{1} \rho_{0}$, then one obtains activation energies in Hartree theory which are identical at $\nu= \pm 1$ and \pm 4 , while a larger energy is predicted at $\nu=0$ [see Eqs. (83), (86), and (87)]. It is intriguing to note that experimentally the quantized Hall plateau at $\nu=0$ sets in at $B \approx 11 \mathrm{~T}$, while the $\nu$ $= \pm 1$ and \pm 4 plateaus are resolved at similar fields around $B \approx 17 \mathrm{~T}$, which may be consistent with the relative magnitudes of the above Hartree energies.

We emphasize that the preceding discussion is by no means intended to be conclusive, but only to suggest one possible mechanism for the appearance of the additional quantum Hall plateaus at high magnetic fields. Again, assessing the origin of the quantum Hall states at $\nu=0$ and $\nu= \pm 1$ requires further experiments, which we hope this work may stimulate. Measuring the activation energies at these filling factors as a function of an in-plane magnetic field may provide some guidance as to whether the quantum Hall states at these filling factors are due to quantum Hall ferromagnetism, explicit symmetry breaking, or perhaps some other mechanism. For instance, if $g \mu_{B} B+2 u_{0} \rho_{0}>4 u_{1} \rho_{0}$, our Hartree estimates predict that the $\nu=0$ gap will increase with an inplane magnetic field component, while the gaps at $\nu= \pm 1$ will remain unchanged. On the other hand, for $g \mu_{B} B$ $+2 u_{0} \rho_{0}<4 u_{1} \rho_{0}$ the gaps at both $\nu=0$ and $\nu= \pm 1$ vary with an increasing in-plane field, the former decreasing while the latter increases.

\section{CONCLUDING REMARKS}

As technological progress enables the fabrication of higher-quality graphene samples, quantum Hall ferromagnetism will likely provide an interesting avenue of exploration along the road to the fractional quantum Hall effect. One of the remarkable aspects of quantum Hall ferromagnetism in graphene is the connection between quantum Hall physics operating on long length scales of order the magnetic length and lattice-scale physics. The clearest manifestation of this interplay occurs at filling factors $\nu= \pm 1$ and possibly also $\nu$ $=0$, where lattice-scale charge density wave order, albeit weak, coexists with the integer quantum Hall effect. Establishing the presence of such lattice-scale structure experimentally would be quite interesting, although doing so will likely prove challenging as the signatures of such order are expected to be small. Another remarkable aspect of quantum Hall ferromagnetism in graphene that would be worth pursuing is the potential for observing a Kosterlitz-Thouless transition, which we argued should occur at filling factors $\nu$ $= \pm 3$ and \pm 5 . Further experimental and theoretical studies of Skyrmion physics in graphene would also be interesting.

In the more immediate future, further experiments to determine the origin of the additional integer quantum Hall states appearing at high magnetic fields would be extremely useful. We have provided here Hartree estimates for the activation energies in these states which, at least in principle, should be straightforward to compare with by exploring the dependence of the transport gap on an in-plane magnetic field. Finally, studies that incorporate disorder into our analysis of the paramagnetic regime in a more systematic way would of course be welcome.

Note added in proof. After submission of this manuscript, a third scenario for the appearance of additional quantum Hall states in graphene at high magnetic fields (apart from quantum Hall ferromagnetism and explicit symmetry breaking) was proposed in Ref. 36 based on the formation of an excitonic gap together with Zeeman splitting. Future experiments will hopefully provide insight into which of these mechanisms underlies the interesting high-field behavior observed in graphene.

\section{ACKNOWLEDGMENTS}

We would like to thank Andrei Bernevig, Taylor Hughes, Allan MacDonald, and especially Leon Balents for stimulating discussions, as well as Philip Kim for sharing experimental data prior to publication. This work was supported by the National Science Foundation through Grants Nos. PHY9907949 (M.P.A.F.) and DMR-0529399 (J.A. and M.P.A.F.).
${ }^{1}$ K. S. Novoselov, A. K. Geim, S. V. Morozov, D. Jiang, Y. Zhang, S. V. Dubonos, I. V. Grigorieva, and A. A. Firsov, Science 306, 666 (2004).

${ }^{2}$ K. S. Novoselov, A. K. Geim, S. V. Morozov, D. Jiang, M. I. Katsnelson, I. V. Grigorieva, S. V. Dubonos, and A. A. Firsov, Nature (London) 438, 197 (2005).
${ }^{3}$ Y. Zhang, Y.-W. Tan, H. L. Stormer, and P. Kim, Nature (London) 438, 201 (2005).

${ }^{4}$ K. S. Novoselov, E. McCann, S. V. Morozov, V. I. Fal'ko, M. I. Katsnelson, U. Zeitler, D. Jiang, F. Schedin, and A. K. Geim, Nat. Phys. 2, 177 (2006).

${ }^{5}$ Y. Zhang, Z. Jiang, J. P. Small, M. S. Purewal, Y.-W. Tan, M. 
Fazlollahi, J. D. Chudow, J. A. Jaszczak, H. L. Stormer, and P. Kim, Phys. Rev. Lett. 96, 136806 (2006).

${ }^{6}$ Y. Zheng and T. Ando, Phys. Rev. B 65, 245420 (2002).

${ }^{7}$ V. P. Gusynin and S. G. Sharapov, Phys. Rev. Lett. 95, 146801 (2005).

${ }^{8}$ N. M. R. Peres, F. Guinea, and A. H. Castro Neto, Phys. Rev. B 73, 125411 (2006).

${ }^{9}$ A. H. Castro Neto, F. Guinea, and N. M. R. Peres, Phys. Rev. B 73, 205408 (2006).

${ }^{10}$ E. McCann and V. I. Fal'ko, Phys. Rev. Lett. 96, 086805 (2006).

${ }^{11}$ D. N. Sheng, L. Sheng, and Z. Y. Weng, Phys. Rev. B 73, 233406 (2006).

${ }^{12}$ L. Brey and H. A. Fertig, Phys. Rev. B 73, 195408 (2006).

${ }^{13}$ D. A. Abanin, P. A. Lee, and L. S. Levitov, Phys. Rev. Lett. 96, 176803 (2006).

${ }^{14}$ V. Lukose, R. Shankar, and G. Baskaran, cond-mat/0603594 (unpublished).

${ }^{15}$ K. Nomura and A. H. MacDonald, Phys. Rev. Lett. 96, 256602 (2006).

${ }^{16}$ H. A. Fertig and L. Brey, cond-mat/0604260 (unpublished).

${ }^{17}$ S. M. Girvin, in The Quantum Hall Effect: Novel Excitations and Broken Symmetries, Proceedings of the Les Houches Summer School of Theoretical Physics, 1998, edited by A. Comtet, T. Jolicoeur, S. Ouvry, and F. David (Springer-Verlag, Berlin, 1999).

${ }^{18}$ S. M. Girvin and A. H. MacDonald, in Perspectives in Quantum Hall Effects: Novel Quantum Liquids in Low-Dimensional Semiconductor Structures, edited by S. Das Sarma and A. Pinczuk (Wiley, New York, 1997).

${ }^{19}$ J. Gonzalez, F. Guinea, and M. A. H. Vozmediano, Phys. Rev. B 59, R2474 (1999).
${ }^{20}$ F. D. M. Haldane, Phys. Rev. Lett. 61, 2015 (1988).

${ }^{21}$ C. L. Kane and E. J. Mele, Phys. Rev. Lett. 95, 226801 (2005).

${ }^{22}$ N. D. Mermin and H. Wagner, Phys. Rev. Lett. 17, 1133 (1966).

${ }^{23}$ D. P. Arovas, A. Karlhede, and D. Lilliehook, Phys. Rev. B 59, 13147 (1999).

${ }^{24}$ S. L. Sondhi, A. Karlhede, S. A. Kivelson, and E. H. Rezayi, Phys. Rev. B 47, 16419 (1993).

${ }^{25}$ X.-G. Wu and S. L. Sondhi, Phys. Rev. B 51, 14725 (1995).

${ }^{26}$ K. Moon, H. Mori, K. Yang, S. M. Girvin, A. H. MacDonald, L. Zheng, D. Yoshioka, and S.-C. Zhang, Phys. Rev. B 51, 5138 (1995).

${ }^{27}$ M. Rasolt, B. I. Halperin, and D. Vanderbilt, Phys. Rev. Lett. 57, 126 (1986)

${ }^{28}$ H. A. Fertig, L. Brey, R. Cote, and A. H. MacDonald, Phys. Rev. B 50, 11018 (1994).

${ }^{29}$ Y. P. Shkolnikov, S. Misra, N. C. Bishop, E. P. De Poortere, and M. Shayegan, Phys. Rev. Lett. 95, 066809 (2005).

${ }^{30}$ T. A. Gloor and F. Mila, Eur. Phys. J. B 38, 9 (2004).

${ }^{31}$ K. Yang, K. Moon, L. Zheng, A. H. MacDonald, S. M. Girvin, D. Yoshioka, and S.-C. Zhang, Phys. Rev. Lett. 72, 732 (1994).

${ }^{32}$ M. M. Fogler and B. I. Shklovskii, Phys. Rev. B 52, 17366 (1995).

${ }^{33}$ J. Sinova, A. H. MacDonald, and S. M. Girvin, Phys. Rev. B 62 , 13579 (2000).

${ }^{34}$ D. R. Leadley, R. J. Nicholas, J. J. Harris, and C. T. Foxon, Phys. Rev. B 58, 13036 (1998).

${ }^{35}$ B. A. Piot, D. K. Maude, M. Henini, Z. R. Wasilewski, K. J. Friedland, R. Hey, K. H. Ploog, A. I. Toropov, R. Airey, and G. Hill, Phys. Rev. B 72, 245325 (2005).

${ }^{36}$ V. P. Gusynin, V. A. Miransky, S. G. Sharapov, and I. A. Shovkovy, cond-mat/0605348 (unpublished). 